\title{
A Pilot Survey of Odontomas for Size Constancy Using Cone-Beam Computed Tomography: Effect of Age, Sex, Lesion Location, and Histological Type: A Case Series
}

\author{
Shinichiro Yamada*, Hizuru Osanai, Katsumitsu Shimada, Hiromasa Hasegawa, Keiichi Uchida \\ Department of Oral and Maxillofacial Radiology, School of Dentistry, Matsumoto Dental University, Shiojiri, Japan \\ Email: ^shinichiro.yamada@mdu.ac.jp, hizuru.osanai@mdu.ac.jp, katsumitsu.shimada@mdu.ac.jp, \\ hiromasa.hasegawa@mdu.ac.jp, keiichi.uchida@mdu.ac.jp
}

How to cite this paper: Yamada, S., Osanai, H., Shimada, K., Hasegawa, H. and Uchida, K. (2021) A Pilot Survey of Odontomas for Size Constancy Using Cone-Beam Computed Tomography: Effect of Age, Sex, Lesion Location, and Histological Type: A Case Series. Open Journal of Stomatology, 11, 467-477.

https://doi.org/10.4236/ojst.2021.1112042

Received: October 7, 2021

Accepted: December 18, 2021

Published: December 21, 2021

Copyright $\odot 2021$ by author(s) and Scientific Research Publishing Inc. This work is licensed under the Creative Commons Attribution International License (CC BY 4.0).

http://creativecommons.org/licenses/by/4.0/ (c) (i) Open Access

\begin{abstract}
Objectives: Odontoma is the most common type of odontogenic tumors. Many studies have analyzed the statistical associations between odontoma location and patient age and sex, according to the histological tumor type. However, few studies have assessed odontoma morphological characteristics using cone-beam computed tomography (CBCT). We aimed to evaluate the association between odontoma location and size. Methods: We performed CBCT on patients with odontomas (19 patients; 10 women, 9 men; average age, 12.6 [range, 6 - 34] years) according to the pathology type at a university hospital between April 2008 and February 2017. The locations of the lesions were noted, and their sizes were measured on CBCT images. Buccolingual, mesiodistal, and vertical diameters of the lesions were recorded on the same slice with the greatest diameters on axial, coronal, and sagittal CBCT images. Results: Altogether, 9 (47.4\%) and 10 (52.6\%) odontomas were located in the mandible and maxilla, respectively. There was no significant difference in the mesiodistal and vertical diameters on the CBCT image between the mandibular and maxillary groups when the odontoma size was compared with location $(p<0.05)$. However, the average diameters in only the buccolingual diameter were significantly greater in the maxilla. There were no significant differences between the two groups according to sex, age, or histological type. Conclusions: These data suggest that the sizes of odontomas in the maxilla are affected by bone expansion in the buccolingual direction, but they may be invariable in most settings. Three-dimensional assessment across the age groups suggests a lack of variation in size.
\end{abstract}




\section{Keywords}

Cone-Beam Computed Tomography (CBCT), Odontoma, Size, Location

\section{Introduction}

Odontomas are generally classified as compound or complex. In radiographic studies, odontomas appear as well-defined lesions with a radiotransparent halo and contain radiodense zones [1] [2] [3]. In general, they are asymptomatic and slow-growing, reaching no more than $3 \mathrm{~cm}$ in diameter [4]. Several statistical evaluations of the association between the odontoma location and the sex and age of patients have been performed [5]. However, few reports have examined the maximum diameter of odontomas with respect to tumor location [6] [7] [8]. Previous studies indicated that some radiographic findings are particularly helpful for the diagnosis of odontomas. However, to the best of our knowledge, no study has ever attempted to analyze the size (vertical diameter) of lesions in this context. Odontomas mostly develop during the first to second decade of life of patients, which may be associated with infection and malocclusion [9] [10]. The management of odontomas is important to maintain appropriate development of dentitions. In children, when an odontoma is $30 \mathrm{~mm}$ or more in size, it occupies a wide area in the mandible and maxilla. Especially when the anterior teeth and mandibular molars are affected, the patient experiences swelling of the mandible and maxilla and facial swelling, which makes facial appearance a concern after excision. In addition to deformity and dysfunction, there is concern about the effect on mandibular growth [11]. Unfortunately, we have poor data on the relationship between tumor size and location. Cone-beam computed tomography (CBCT) can easily help us determine the three-dimensional size of odontomas. Therefore, the maximum diameters of odontomas and their association with their location were examined using $\mathrm{CBCT}$ to obtain data for the management of odontomas.

\section{Materials and Methods}

This retrospective study included 19 patients with a confirmed diagnosis of odontoma on preoperative imaging examination at a university hospital between April 2008 and February 2017, where inspection using CBCT was a prerequisite. Patients with incomplete data, such as missing pathology examination findings, were excluded. There were 14 cases of compound odontoma and 5 cases of complex odontoma confirmed on pathological examination. There were 10 women (52.6\%) and 9 men (47.4\%), with an average age (standard deviation [SD]) of 11.3 (8.7) (range, 6 - 34) years.

A CBCT device (3D Accuitomo F17 ${ }^{\circledR}$, Morita Corporation, Kyoto) was used to capture images of the odontoma in each patient. The following imaging parameters were used: slice thickness, $0.5 \mathrm{~mm}$; tube voltage, $80-90 \mathrm{kV}$; tube cur- 
rent, 5 - $8 \mathrm{~mA}$; voxel size, $0.25 \mathrm{~mm}$; scan time, $20 \mathrm{~s}$; and field of view, $40 \times 40$ $100 \times 100 \mathrm{~mm}$. We reconstituted the volume data using viewer software (i-VIEW; Hologic Inc., Marlborough, Massachusetts, USA) and reobserved the sliced images following CBCT imaging. The reading shadow of the CBCT image and the measurements were similar according to the high-definition monitor on the electronic chart. Odontomas were classified into four categories with respect to the location of the tumor: the mandibular and maxillary anterior tooth regions and posterior tooth regions.

All measurements were acquired with respect to the occlusal plane. The diameter was measured in the mandibular cortical bone through a perpendicular line drawn from the base of the inferior border of the mandible or midsection of the palatine bone, which formed a mesiodistal diameter along the dentition and the buccolingual diameter, perpendicular to the dentition. The diameter of the mandibular and maxillary lesions was measured, where the greatest size of the odontoma was seen in images in the axial, coronal, and sagittal planes (Figure 1). Two investigators (oral radiologists) were trained for tumor measurement using $10 \%$ of the cases. They then independently measured the odontoma in each case. For cases in which there was a disagreement on tumor size, a consensus was achieved by discussion. Among the 19 cases, 14 were determined as compound odontomas and 5 as complex odontomas.

Three measurements were recorded for each patient, and the average of the obtained values was used to minimize errors. An analysis of variance (ANOVA) was applied to evaluate the differences in the greatest and average odontomas in the buccolingual, mesiodistal, and vertical diameters between odontomas located in the mandibular and maxillary regions, mean difference according to the sex of the patient, and mean difference according to the histological type on CBCT imaging.

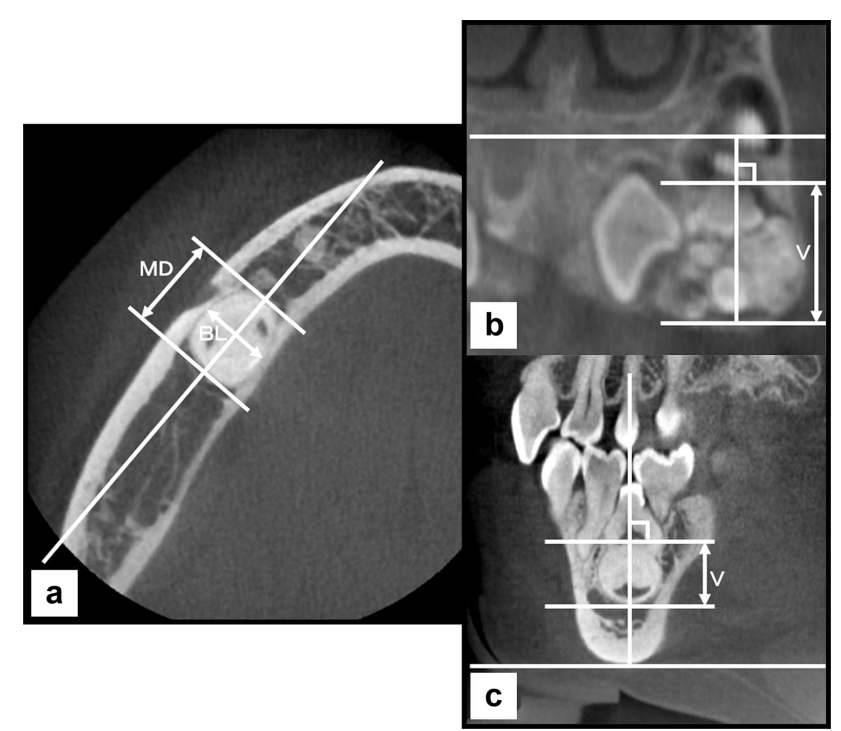

Figure 1. Morphological measurement of the odontoma lesions using three-dimensional cone-beam computed tomography images. (a) Axial plane. (b) Coronal plane. (c) Sagittal plane. $M D$ mesiodistal diameter, $B L$ buccolingual diameter, $V$ vertical diameter. 
Statistically, all values were assessed with the Shapiro-Wilk normality and Bartlett tests for numeric data prior to evaluation. A two-sample t-test and one-way ANOVA were used for testing the differences among the groups. Data are presented as average (SD) or frequency (\%). Differences were considered statistically significant when $p$ values were $<0.05$.

\section{Results}

Odontomas were diagnosed on panoramic radiographs and underwent further detailed evaluation using CBCT. Nineteen lesions were located in the mandible in $10(52.6 \%)$ lesions and the maxilla in 9 (47.4\%) lesions. The group with maxillary lesions consisted of four $(21.1 \%)$ women and five $(26.3 \%)$ men, while the group with mandibular lesions consisted of five women $(26.3 \%)$ and five men (26.3\%). The average age at the first medical examination was 12.6 (range, 6 $34)$ years; 13 patients $(68.4 \%)$ were 11 years or older, while $6(31.6 \%)$ were 10 years old or younger. When the location of the lesion was classified according to the anterior tooth regions and posterior tooth regions, the mandibular lesions included five in the mandibular anterior tooth region and five in the mandibular posterior tooth region, while seven lesions were found in the maxillary anterior tooth region, and two lesions were found in the maxillary posterior tooth region. All 19 odontomas were less than $30 \mathrm{~mm}$ in the buccolingual, mesiodistal, and vertical diameters, and most presented with a size of approximately $10 \mathrm{~mm}$.

\subsection{Comparison of Quadrant Part Sizes in the Mandibular and Maxilla Regions}

In the mandible, the buccolingual diameter of the lesions was $6.0-14.4 \mathrm{~mm}$, with a buccolingual diameter of $9.04(\mathrm{SD}, 1.90) \mathrm{mm}$. The size of the lesions in the buccolingual diameter in the maxilla was significantly larger than that in the mandible $(p<0.05)$. In the maxilla, the buccolingual diameter of the lesions on the CBCT image of the odontoma was $6.3-15.1 \mathrm{~mm}$, with a diameter of 11.09 (SD, 2.32) $\mathrm{mm}$ (Figure 2(a)). These findings in the mandibular and maxillary anterior and canine-posterior regions were not significantly different. Figure 3 shows that there were no significant differences among the lesion extensions in the three diameters.

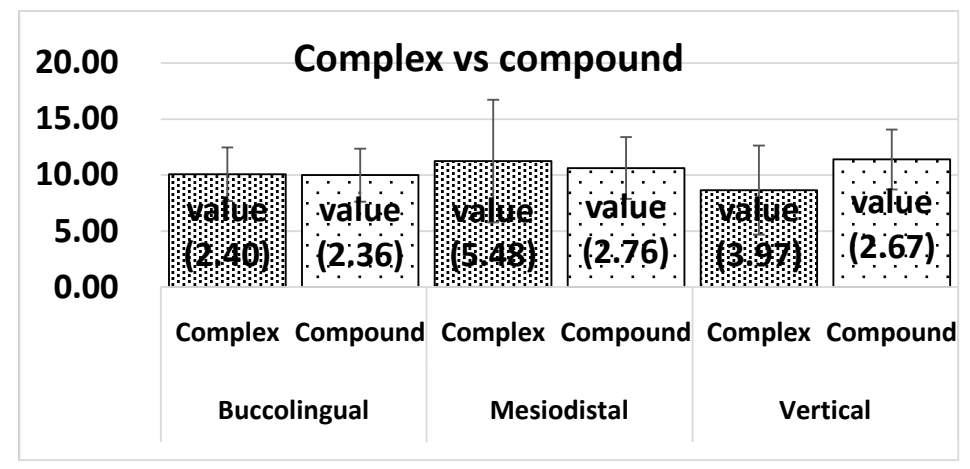

(a) 


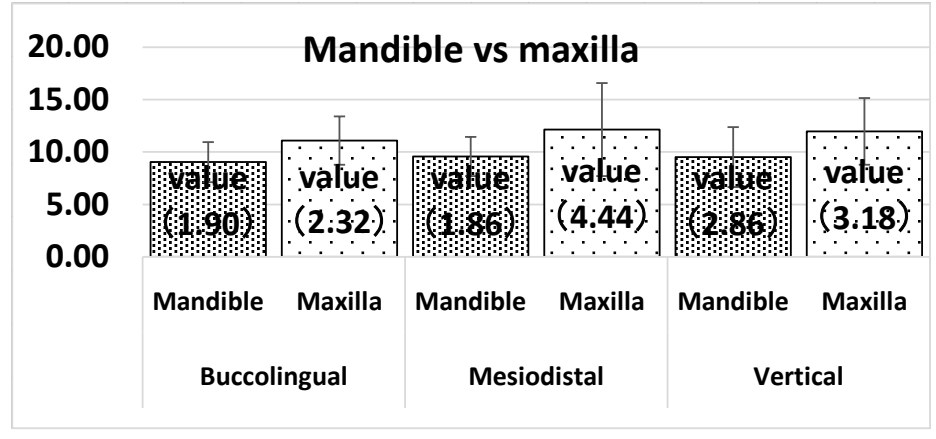

(b)

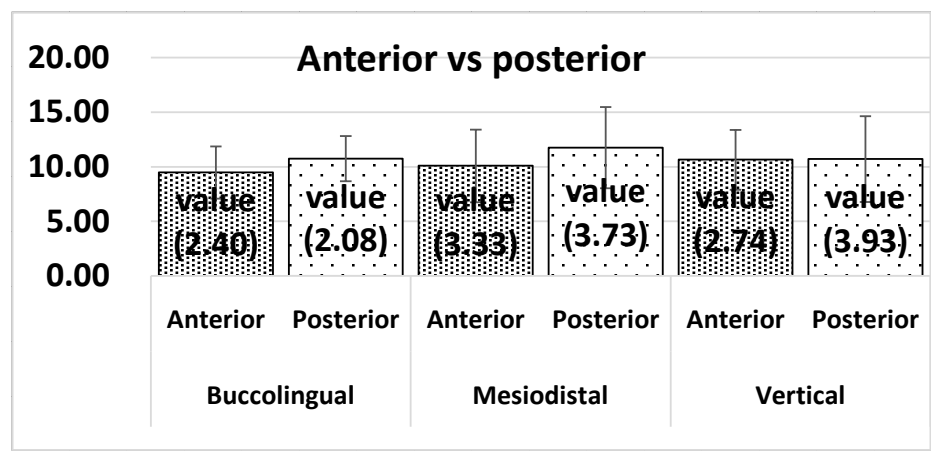

(c)

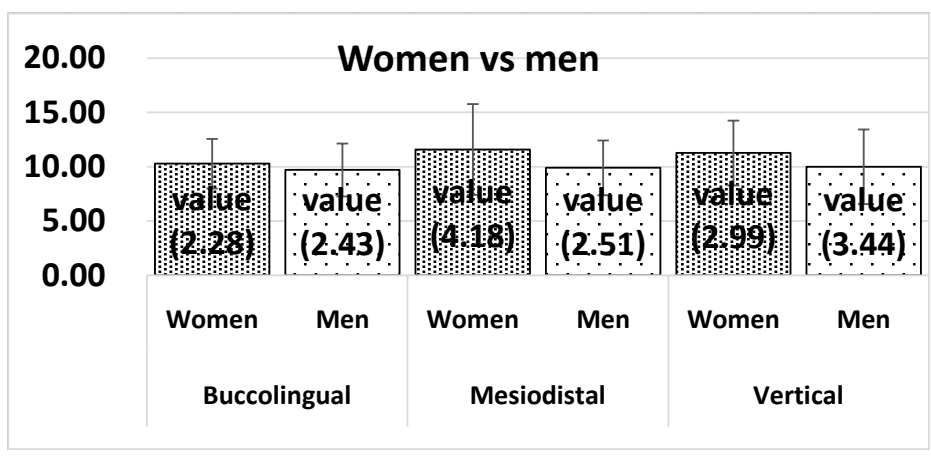

(d)

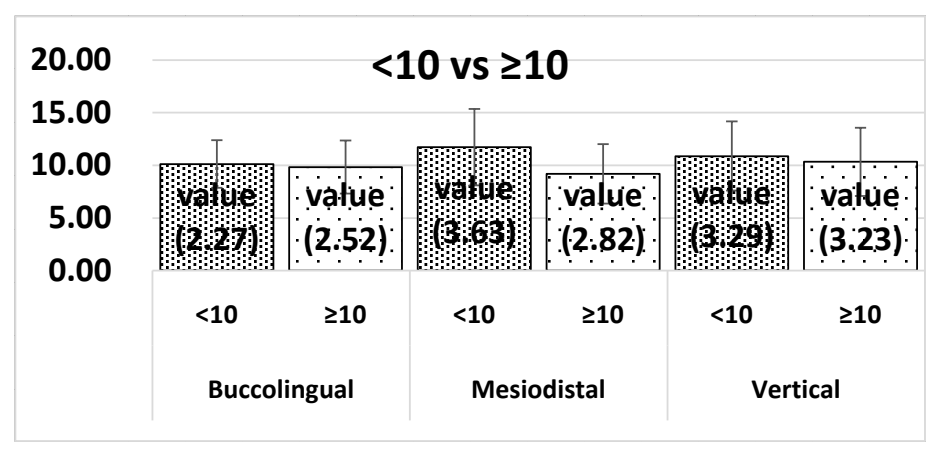

(e)

Figure 2. Comparison of lesion sizes by cone-beam computed tomography images. (a) Comparison of lesion sizes in the mandible and maxilla. (b) Comparison of lesion sizes in the mandibular and maxillary anterior and posterior tooth regions. (c) Comparison of lesion sizes between the sexes. (d) Comparison of lesion sizes between ages $<10$ and $\geq 10$ years. (e) Comparison of lesion sizes of complex and compound odontomas. 
a

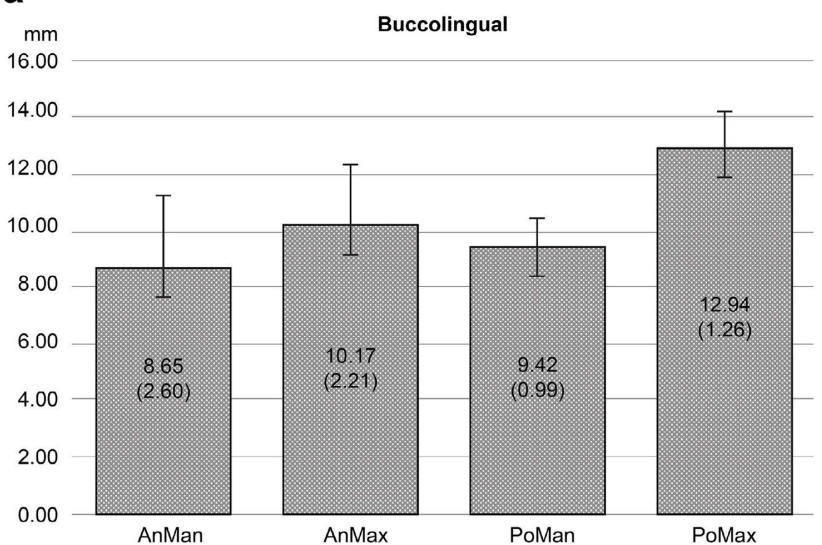

$\mathbf{C}_{\mathrm{mm}} \quad$ Vertical

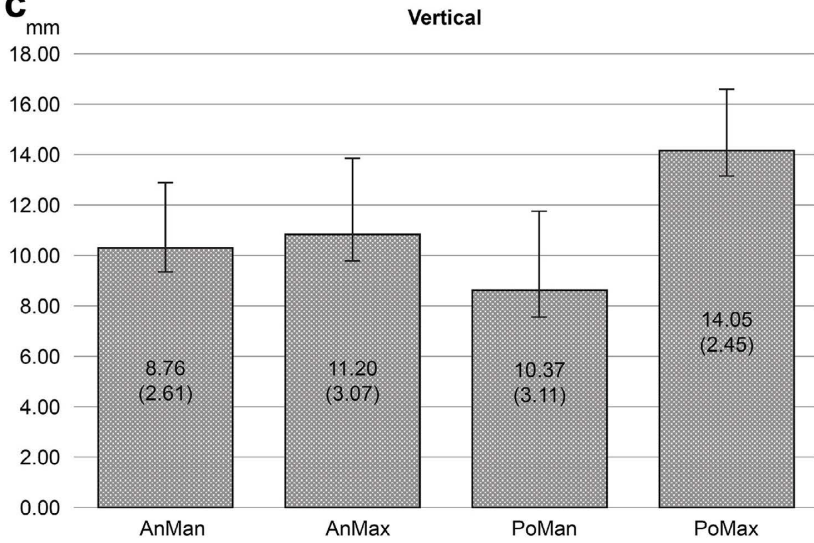

b

Mesiodistal

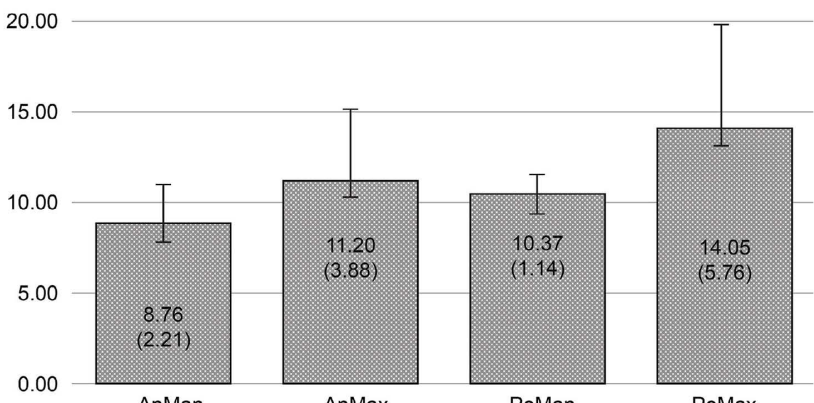

AnMan

AnMax

PoMan

PoMax

Figure 3. Comparison of quadrant lesion sizes in the mandibular and maxillary anterior and posterior tooth regions. AnMan mandibular anterior tooth, AnMax maxillary anterior tooth, Poman mandibular posterior tooth, PoMax maxillary posterior tooth.

\subsection{Comparison of Size in the Maxillary and Mandibular Anterior Tooth Regions and Posterior Tooth Regions}

The average diameter of the odontoma in the anterior and posterior tooth regions in each patient was compared. Figure 2 (b) shows the detailed results of the size of the lesions in the maxillary anterior and posterior tooth regions, compared with the size of the lesions in the mandibular anterior and posterior tooth regions, as seen on the $\mathrm{CBCT}$ images.

\subsection{Comparison of Size between Sexes, between Ages $<10$ and $\geq 10$ Years, and between Complex and Compound Odontomas}

Figure 2(c) shows that there were no significant differences between ages $<10$ and $\geq 10$ years and the lesion extensions in the three diameters. Figure 2(d) shows the diameters of the greatest odontoma based on the patient's sex and a detailed comparison of the size of the odontomas on СBCT images.

Table 1 shows the frequency of lesion occurrence with respect to the location of complex and compound odontomas. The frequency of occurrence of odontomas was categorized by site and type; compound odontomas were found in five cases $(26.3 \%)$ in the mandibular and maxillary anterior tooth regions, and complex odontomas were found in three cases (15.8\%) in the mandibular posterior 
Table 1. Frequency of lesion occurrence with respect to the location of the complex and compound odontomas.

\begin{tabular}{cccc}
\hline & $\begin{array}{c}\text { Anterior tooth } \\
\text { region }\end{array}$ & $\begin{array}{c}\text { Posterior tooth } \\
\text { region }\end{array}$ & Total \\
\hline Maxillary (complex odontoma) & 1 & 1 & 2 \\
(Compound odontoma) & 5 & 2 & 7 \\
Mandibular (complex odontoma) & 0 & 3 & 3 \\
(Compound odontoma) & 5 & 2 & 7 \\
Total & 11 & 8 & 19 \\
\hline
\end{tabular}

tooth regions. The diameter of the complex and compound lesions is shown in Figure 2(e). No significant difference in size based on sex and type of odontoma (complex and compound) was found $(p<0.05)$.

\subsection{Comparison of Size between Quadrant Locations}

Figures 3(a)-3(c) shows the four numeric locations and sizes of each odontoma. In the maxillary posterior tooth regions, the buccolingual diameter of the lesions was larger than that of the mandible, but the difference was not statistically significant. Within each location, the three measurements were similar in both mandible and maxilla. The effects of the lesion extensions on odontoma morphology were evaluated independently in the odontomas.

\section{Discussion}

The knowledge of the epidemiology characteristics such as anatomic location, age, and sex of the patients with odontoma is extremely valuable in the development of diagnoses. However, the three measurements of the radiographic features of their mandibular and maxillary entities are scarcely described. Our findings regarding tumor location were consistent with those of previous reports [12] [13] [14] [15] [16]. The average age (12.6 years) and sex distribution of patients with odontoma (9:10) in this study are mostly consistent with the literature [17]. Several reports have stated that compound odontomas appear more frequently than complex odontomas [12] [18] [19] [20], which corroborates the findings of our study. Moreover, previous reports stated that odontomas showed significant differences according to the sex of the patient and type of odontoma [20] [21], but no differences in sex were observed in our study. In terms of the frequency of occurrence by site and type, compound odontomas were mainly found in the posterior mandibular area [22]. However, in the present study, there were no characteristic findings besides an abundance of compound odontomas in the mandible and maxilla.

To the best of our knowledge, the present clinical study demonstrated for the first time a precise assessment of the odontoma size characteristic for patient obtained from CBCT images. Evaluating the ratio of the three diameters, an obtained value was helpful in the assessment of a shape of odontoma. Interestingly, we observed that the findings from this investigation indicate that the differenc- 
es between the three diameters are of small magnitude and of no statistical significance; there is no variation from $\mathrm{CBCT}$ in the buccolingual, mesiodistal, and vertical diameters of the lesions among the quadrant locations of the mandible and maxilla. Odontomas occurring in the maxilla are accompanied by an enlargement of the buccolingual diameter, and those that occur in the mandible progress without variation along the buccolingual, mesiodistal and vertical dimensions. This may reflect as changes in the size of spherical lesions. This finding has not been described in previous studies.

To our knowledge, compared with mandibular lesions, maxillary lesions have limited space for growth owing to the buccal and lingual firm cortical bone. Therefore, the difference between the growth pattern of mandibular and maxillary odontomas may be partly due to the greater cortical thickness of the mandible compared with that of the maxilla [23]. With the increase in size, many large lesions in the mandible and maxilla have a circular or oval radiographic appearance with cortical expansion and cortical bone thinning, loss of bony continuity, and root resorption of at least one adjacent tooth [24]. This may have been the reason why the sizes of the odontomas depended on the size of the mandible itself.

There were no significant differences between women and men with respect to the buccolingual, mesiodistal, and vertical diameters of the mandible and maxilla. Thus, the size of the odontoma was not related to the size of the mandible and maxilla with respect to sex, and it was considered that the growth process did not affect tumor growth.

Considerable controversy exists regarding age distribution. Studies on odontomas include patients of all ages [20]. Other studies point to a higher incidence of odontomas in the first decades of life [7] [25]. The relationship between the patient age, location of the odontoma, and three-dimensional size of the odontoma remains unclear. Some studies have found that it is possible for patient age to be one of the most important factors in the growth process of an odontoma [26]. Comparisons of location as well as sums of groups of odontomas were performed between women and men within and between ages $<10$ and $\geq 10$ years. The three-dimensional sizes of the odontomas had a similar trend in the two age groups examined in the present study, which may indicate that the size of odontoma was relatively invariable at all ages.

The size of the odontomas did not change significantly with normal aging and location. On average, most odontomas grow up to approximately $10 \mathrm{~mm}$, and the size of each lesion varies. These findings are consistent with previous literature that has described small-sized odontoma (smaller than the impacted permanent tooth) [7]. The size of the lesion did not create the need for marsupialization before removal, and there was a satisfactory amount of bone surrounding the lesion. Instead, the misdiagnosis or failure to diagnose can lead to aesthetic and phonetic disorders, with progression until drastic occlusal changes occur [27]. 


\section{Conclusion}

In summary, odontomas in the maxilla tend to be circular or oval and grow with buccal dimension, and it seems that there is a single pattern of the odontoma size. This makes a precise assessment of the odontoma size characteristic for patient as very helpful in clinical dental practice. The determination of the shape, size, and location of the odontoma is very important taking into consideration numerous dental procedures carried out in the mandible and maxilla. Thus, obtained results could be very useful for many clinicians. However, the limitation of this study is that we do not know the clinical truth for the individual odontomas. We could not determine the accuracy of any of the individual measurements. Further, the sample size of this study was relatively small $(n=19)$.

\section{Conflicts of Interest}

The authors declare no conflicts of interest regarding the publication of this paper.

\section{References}

[1] Lucas, R.B. (1984) Odontoma, Pathology of Tumours of the Oral Tissue. 4th Edition, Churchill Livingstone, London.

[2] Gibilisco, J.A. (1985) Stafne's Oral Radiographic Diagnosis. 5th Edition, W.B. Saunders Company, Philadelphia.

[3] El-Naggar, A.K., Chan, J.K.C., Grandis, J.R., Takata, T. and Slootweg, P.J. (2017) WHO Classification of Head and Neck Tumours. 4th Edition, IARC Press, Lyon.

[4] Sanchez, O.H., Leco-Berrocal, M.I. and Martínez-González, J.M. (2008) Metaanalysis of the Epidemiology and Clinical Manifestations of Odontomas. Medicina Oral, Patologia Oral, Cirugia Bucal, 13, 730-734.

[5] Siriwardena, B.S.M.S., Crane, H., O’Neill, N., Abdelkarim, R., Brierley, D.J., Franklin, C.D., et al. (2019) Odontogenic Tumors and Lesions Treated in a Single Specialist Oral and Maxillofacial Pathology Unit in the United Kingdom in 1992-2016. Oral Surgery, Oral Medicine, Oral Pathology and Oral Radiology, 127, 151-166. https://doi.org/10.1016/j.0ooo.2018.09.011

[6] Miki, Y., Oda, Y., Iwaya, N., Hirota, M., Yamada, N., Aisaki, K., et al. (1999) Clinicopathological Studies of Odontoma in 47 Patients. Journal of Oral Science, 41, 173-176. https://doi.org/10.2334/josnusd.41.173

[7] Levi-Duque, F. and Ardila, C.M. (2019) Association between Odontoma Size, Age and Gender: Multivariate Analysis of Retrospective Data. Journal of Clinical and Experimental Dentistry, 11, 701-706. https://doi.org/10.4317/jced.55733

[8] Buyukcavus, M.H., Satir, S. and Ozei, S. (2020) Evaluation of Dentofacial Asymmetry Caused by Odontomas Using Panoramic Radiographs. Nigerian Journal of Clinical Practice, 23, 291-297.

[9] Altay, M.A., Ozgur, B. and Cehreli, Z.C. (2016) Management of a Compound Odontoma in the Primary Dentition. Journal of Dentistry for Children (Chic), 83, 98-101.

[10] Tamassoki, S., Khosravi, M. and Azizi, F. (2017) Timing of Surgical Removal of Odontoma in a Growing Orthodontic Patient: A Case Report. International Journal of Orthodontics, 28, 49-52. 
[11] Gorlin, R.J., Chaudhry, A.P. and Pindborg, J.J. (1961) Odontogenic Tumors. Classification, Histopathology, and Clinical Behavior in Man and Domesticated Animals. Cancer, 14, 73-101. https://doi.org/10.1002/1097-0142(196101/02)14:1<73::AID-CNCR2820140111>3.0. CO;2-T

[12] Regezi, J.A., Kerr, D.A. and Courtney, R.M. (1978) Odontogenic Tumors: Analysis of 706 Cases. International Journal of Oral Surgery, 36, 771-778.

[13] Fernandes, A.M., Duarte, E.C., Pimenta, F.J., Souza, L.N., Santos, V.R., Mesquita, R.A., et al. (2005) Odontogenic Tumors: A Study of 340 Cases in a Brazilian Population. Journal of Oral Pathology \& Medicine, 34, 583-587. https://doi.org/10.1111/j.1600-0714.2005.00357.x

[14] Kaugars, G.E., Miller, M.E. and Abbey, L.M. (1989) Odontomas. Oral Surgery, Oral Medicine, Oral Pathology and Oral Radiology, 67, 172-176. https://doi.org/10.1016/0030-4220(89)90324-1

[15] Yadav, M., Godge, P., Meghana, S.M. and Kulkarni, S.R. (2012) Compound Odontoma. Contemporary Clinical Dentistry, 3, 13-15. https://doi.org/10.4103/0976-237X.95095

[16] Cohen, D.M. and Bhattacharyya, I. (2004) Ameloblastic Fibroma, Ameloblastic Fibro-Odontoma, and Odontoma. Oral and Maxillofacial Surgery Clinics of North America, 16, 375-384. https://doi.org/10.1016/j.coms.2004.03.005

[17] Soluk Tekkesin, M., Tuna, E.B., Olgac, V., Aksakall, N. and Alatll, C. (2016) Odontogenic Lesions in a Pediatric Population: Review of the Literature and Presentation of 745 Cases. International Journal of Pediatric Otorhinolaryngology, 86, 196-199. https://doi.org/10.1016/j.ijporl.2016.05.010

[18] Or, S. and Yucetas, S. (1987) Compound and Complex Odontomas. International Journal of Oral and Maxillofacial Surgery, 16, 596-599. https://doi.org/10.1016/S0901-5027(87)80112-1

[19] Owens, B.M., Schuman, N.J., Mincer, H.H., Turner, J.E. and Oliver, F.M. (1997) Dental Odontomas: A Retrospective Study of 104 Cases. Journal of Clinical Pediatric Dentistry, 21, 261-264.

[20] Hidalgo-Sanchez, O., Leco-Berrocal, M.I. and Martínez-Gonzalez, J.M. (2008) Metaanalysis of the Epidemiology and Clinical Manifestations of Odontomas. Medicina Oral, Patologia Oral, Cirugia Bucal, 13, 730-734.

[21] Hisatomi, M., Asaumi, J.I., Konouchi, H., Honda, Y., Wakasa, T. and Kishi, K (2002) A Case of Complex Odontoma Associated with an Impacted Lower Deciduous Second Molar and Analysis of the 107 Odontomas. Oral Diseases, 8, 100-105. https://doi.org/10.1034/j.1601-0825.2002.1c778.x

[22] Amado Cuesta, S., Gargallo Albiol, J., Berini Aytés, L. and Gay Escoda, C. (2003) Review of 61 Cases of Odontoma. Presentation of an Erupted Complex Odontoma. Medicina Oral, 8, 366-373.

[23] MacDonald, D. (2016) Lesions of the Jaws Presenting as Radiolucencies on Cone-Beam CT. Clinical Radiology, 71, 972-985. https://doi.org/10.1016/j.crad.2016.05.018

[24] Theodorou, S.J., Theodorou, D.J. and Sartoris, D.J. (2007) Imaging Characteristics of Neoplasms and Other Lesions of the Jawbones: Part 1. Odontogenic Tumors and Tumorlike Lesions. Clinical Imaging, 31, 114-119. https://doi.org/10.1016/j.clinimag.2006.12.022

[25] Iatrou, I., Vardas, E., Theologie-Lygidakis, N. and Leventis, M. (2010) A Retrospective Analysis of the Characteristics, Treatment and Follow-Up of 26 Odontomas in 
Greek Children. Journal of Oral Science, 52, 439-447.

https://doi.org/10.2334/josnusd.52.439

[26] Perumal, C.J., Mohamed, A., Singh, A. and Noffke, C.E. (2013) Sequestrating Giant Complex Odontoma: A Case Report and Review of the Literature. Journal of Maxillofacial and Oral Surgery, 12, 480-484. https://doi.org/10.1007/s12663-010-0148-y

[27] Veis, A., Tziafas, D. and Lambrianidis, T. (2000) A Case Report of a Compound Odontoma Causing Delayed Eruption of a Central Maxillary Incisor: Clinical and Microscopic Evaluation. Journal of Endodontics, 26, 477-479.

https://doi.org/10.1097/00004770-200008000-00012 